\title{
Correction to: Using the Goal Attainment Scale adapted for depression to better understand treatment outcomes in patients with major depressive disorder switching to vortioxetine: a phase 4, single-arm, open- label, multicenter study
}

Maggie McCue ${ }^{1 *}$, Sara Sarkey ${ }^{1}$, Anna Eramo², Clement François ${ }^{2}$ and Sagar V. Parikh ${ }^{3}$

Correction to: BMC Psychiatry 21, 622 (2021)

http://orcid.org/10.1186/s12888-021-03608-1

Following publication of the original article [1], the authors identified errors in the text. Below is a table of corrections which have been implemented in the original article.

\begin{tabular}{lll}
\hline Section & $\begin{array}{l}\text { Originally published } \\
\text { text }\end{array}$ & Corrected text \\
Abstract & Treatment response & Treatment response and \\
& and remission rates & remission rates were \\
& were 65 and $40 \%$, & approximately 65 and \\
& respectively. & $40 \%$, respectively. \\
\hline
\end{tabular}

The original article can be found online at https://doi.org/10.1186/s12888021-03608-1.

\section{*Correspondence: Maggie.McCue@takeda.com}

${ }^{1}$ Takeda Pharmaceuticals U.S.A., Inc., 95 Hayden Avenue, Lexington, MA 02421, USA

Full list of author information is available at the end of the article

\begin{tabular}{|c|c|c|}
\hline $\begin{array}{l}\text { Results - Baseline } \\
\text { characteristics }\end{array}$ & $\begin{array}{l}\text { Overall, study } \\
\text { participants were } \\
\text { predominantly white } \\
(69.2 \%) \text {, female } \\
(82.8 \%) \text {, and } \leq 55 \text { years } \\
\text { of age }(77.9 \%) \text {. Mean } \\
\text { PHQ-9 score was } 15.7 \\
\text { (moderately severe } \\
\text { depression), and } \\
59.5 \% \text { of the patients } \\
\text { were employed. }\end{array}$ & $\begin{array}{l}\text { Overall, study partici- } \\
\text { pants were predom- } \\
\text { inantly white }(69.2 \%), \\
\text { female }(82.8 \%), \text { and } \leq 55 \\
\text { years of age }(77.9 \%) \text {. } \\
\text { Mean PHQ-9 score was } \\
15.7 \text { (moderately severe } \\
\text { depression), and } 58.3 \% \\
\text { of the patients were } \\
\text { employed. }\end{array}$ \\
\hline $\begin{array}{l}\text { Results - Safety and } \\
\text { tolerability }\end{array}$ & $\begin{array}{l}\text { Overall, } 117 \text { AEs } \\
\text { were reported by } 59 \\
\text { patients. }\end{array}$ & $\begin{array}{l}\text { Overall, } 117 \mathrm{AEs} \\
\text { deemed related to } \\
\text { study treatment were } \\
\text { reported by } 59 \text { patients. }\end{array}$ \\
\hline $\begin{array}{l}\text { Availability of Data } \\
\text { and Materials }\end{array}$ & $\begin{array}{l}\text { The Goal Attainment } \\
\text { Scale adapted for } \\
\text { depression (Global } \\
\text { Attainment Scale- } \\
\text { Depression } \odot 2021 \\
\text { Takeda Pharmaceu- } \\
\text { ticals U.S.A., Inc. All } \\
\text { rights reserved) }\end{array}$ & $\begin{array}{l}\text { The Goal Attainment } \\
\text { Scale adapted for } \\
\text { depression (Goal Attain- } \\
\text { ment Scale - Depres- } \\
\text { sion @ } 2021 \text { Takeda } \\
\text { Pharmaceuticals U.S.A., } \\
\text { Inc. All rights reserved) }\end{array}$ \\
\hline
\end{tabular}

The original article [1] has been corrected.

\section{Author details}

${ }^{1}$ Takeda Pharmaceuticals U.S.A., Inc., 95 Hayden Avenue, Lexington, MA 02421, USA. ${ }^{2}$ Lundbeck LLC, 6 Parkway North Blvd, Deerfeld, IL 60015, USA. ${ }^{3}$ University of Michigan Health, 1500 E. Medical Center Dr, Ann Arbor, MI 48109, USA. 
Published online: 07 March 2022

\section{Reference}

1. McCue M, et al. Using the Goal Attainment Scale adapted for depression to better understand treatment outcomes in patients with major depressive disorder switching to vortioxetine: a phase 4, single-arm, open-label, multicenter study. BMC Psychiatry. 2021;21:622. https://doi.org/10.1186/ s12888-021-03608-1.

- fast, convenient online submission

- thorough peer review by experienced researchers in your field

- rapid publication on acceptance

- support for research data, including large and complex data types

- gold Open Access which fosters wider collaboration and increased citations

- maximum visibility for your research: over 100M website views per year

At BMC, research is always in progress.

Learn more biomedcentral.com/submissions 